\title{
Tools for the Clinician: The Essentials of Bedside (ED or ICU) Ultrasound for Deep Vein Thrombosis
}

\author{
Joseph Anton Zitek • Jamie Baydoun • \\ Jeremy Baird
}

Published online: 13 April 2013

(C) Springer Science+Business Media New York 2013

\begin{abstract}
Deep vein thrombosis (DVT) is a serious condition that is difficult to diagnose clinically. Venography has long been held as the gold standard for diagnosis of DVT. Ultrasonography has a sensitivity and specificity that approaches that of venography, and has nearly replaced venography for the diagnosis of DVT. Traditionally DVT ultrasounds have been performed by technologists and interpreted by radiologists. These tests generally consist of full leg compression and Doppler studies, which may take over $15 \mathrm{~min}$ to perform. However, several recent studies have demonstrated that simplified versions of these ultrasound protocols, such as two-point compression or threepoint compression, can be performed at the bedside with sensitivities and specificities that are comparable to those of the more comprehensive formal studies. These studies allow emergency medicine and critical care physicians to rapidly diagnose DVT at the bedside, and some studies suggest that these bedside studies are as accurate as formal studies.
\end{abstract}

Keywords DVT · Deep vein thrombosis · Compression ultrasound · Two-point compression . Emergency medicine $\cdot$ Bedside ultrasound

\footnotetext{
J. A. Zitek · J. Baydoun · J. Baird ( $\square)$

USA

e-mail: jbaird@atsu.edu

J. A. Zitek

e-mail: jzitek@medicine.nevada.edu

J. Baydoun

e-mail: meadejamie@gmail.com
}

901 Rancho Lane, Suite 135, Las Vegas, NV 89106,

\section{Introduction}

Deep vein thrombosis (DVT) is a common condition that has significant morbidity and mortality. In the United States, the age-adjusted incidence rate for DVT is over one person per 1000 [1]. A portion of DVTs will propagate to the lung, which can lead to a mortality rate of up to $35 \%$ if the pulmonary embolism goes untreated $[2,3]$. The firstline treatment of DVTs is anticoagulation, which can put patients at risk for complications from bleeding episodes. Given the risks of either thrombus propagation or the risk of bleeding associated with unnecessary treatment, it is paramount to make an accurate and timely diagnosis of DVT.

A meta-analysis from 2005 concluded that individual clinical factors were of limited value in diagnosing DVT [4]. Strategies combining clinical features with D-dimer testing have been shown to decrease the need for formal ultrasound testing; however, in those higher risk patients or those patients in whom a D-dimer screen is positive, imaging is necessary to safely rule out the disease [5].

The gold standard imaging test for DVT is contrast venography [6]. However, contrast venography is invasive, painful, and time-consuming. Several studies have compared deep vein ultrasound to venography and found that both the sensitivity and specificity of ultrasound are greater than $90 \%$, and may approach $100 \%$ [7, 8, 9]. Thus, ultrasound is now the first choice in terms of imaging test for the diagnosis of DVT.

\section{Why do Bedside Ultrasound for DVT?}

Many institutions lack full-time ultrasound technicians, and so diagnosis of DVT during nighttime or weekend hours may 
be delayed. Physicians who work in these situations may be left with the choice of delaying the patient's diagnosis or anticoagulating the patient without a definitive diagnosis. Neither option provides optimal care for the patient.

A bedside DVT ultrasound can be completed quickly, in most cases in just a few minutes, and is usually easy to perform. Meanwhile, sending a patient for a formal DVT ultrasound may delay his or her diagnosis by hours [10•, 11]. Thus, even for those physicians who work in hospitals with around-the-clock availability of ultrasound technicians, bedside DVT ultrasound can decrease the length of patient stay in the emergency department, and decrease the time to diagnosis of DVT.

\section{The Evidence in Favor of Bedside Ultrasound for DVT}

There have been several articles published evaluating the accuracy of emergency medicine and intensive care physician-preformed bedside DVT ultrasounds.

Two-point and three-point vein compression technique are the leading methods used to perform a bedside ultrasound. The two-point compression method has a sensitivity that ranges from 89 to $100 \%[12,13,14 \bullet \cdot]$. A recent study by Crisp et al. used 47 physicians, including emergency medicine attendings, emergency medicine residents, and residents rotating in the $\mathrm{ED}$ from other services who received a 10-minute DVT ultrasound training session. It found that bedside ultrasound for DVT using the two-point compression technique produced a sensitivity of $100 \%$ and a specificity of $99 \%$ [14••]. With these numbers, one might argue that bedside ultrasound could replace formal studies for DVT. However, these results may be overly optimistic, as this study used a convenience sample of patients that may have avoided the patients in whom ultrasound may have been difficult. This would include obese patients or those patients with indwelling central femoral lines. [14••].

A study by Kline et al. [15] evaluating the three-point compression technique performed by a group of emergency physicians resulted in $70 \%$ sensitivity and $89 \%$ specificity in detecting a DVT when compared to technician-performed and radiology-interpreted ultrasonography. These numbers are less promising than the Crisp study results described above, but this study also found that the sensitivity became $100 \%$ for clinicians who had enrolled at least three previous patients.

Another study performed a multicenter, retrospective review comparing 128 intensivist-performed bedside compression DVT ultrasound studies to formal studies performed by technicians and interpreted by radiologists. These authors found that ultrasound imaging performed by the intensivists had a sensitivity of $86 \%$ and a specificity of $96 \%[10 \bullet]$.
Overall there is great potential for bedside sonography to assess for DVT in the acute setting. A systematic review of articles pertaining to emergency physician preformed ultrasound for evaluation of DVT, which included various ultrasound techniques, showed an overall sensitivity of $95 \%$ (95\% CI of 87-99\%) and specificity of $96 \%(95 \%$ CI of 87-99\%) [16]. Although ultrasound is an operatordependent mode of imaging, once operator procedural competency is achieved, bedside ultrasonography for DVT seems to be nearly as accurate as technician-performed and radiology-interpreted DVT ultrasound.

\section{Review of Anatomy}

There are five important deep veins in the leg: two above the calf and three below. From proximal to distal, the first deep vein is the common femoral vein, also known as simply the femoral vein. It originates just distal to the inguinal ligament and quickly gives off an important superficial branch called the great saphenous vein [17]. The common femoral vein next gives off a branch called the deep femoral vein, which can be difficult to evaluate with ultrasound.

Some people use the term "superficial femoral vein" to describe the portion of the femoral vein that is distal to the branching of the deep femoral vein. However, this term is not recommended because the "superficial femoral vein" is an actual deep vein, and despite the confusing terminology, patients with blood clots in this vein will need to be anticoagulated. We prefer to continue to refer the portion of the femoral vein distal to the deep femoral vein branch simply as "the femoral vein".

The femoral vein then courses inferiorly and medially into the adductor canal. It then passes posterior to the knee where it becomes the second major deep vein above the calf, the popliteal vein. The popliteal vein splits into the three deep veins at the level of the calf, the anterior tibial vein, the posterior tibial vein, and the peroneal vein [17]. A thrombus in the femoral vein or popliteal vein results in what is considered a deep vein thrombosis. A thrombus in the calf veins is of questionable clinical significance. Calf vein thrombosis will be further discussed later.

\section{How to do a DVT Ultrasound at the Bedside}

\section{Basics}

A bedside DVT ultrasound is best performed using a linear probe, and studies show that a high frequency $7.5 \mathrm{MHz}$ probe produces excellent results [14••]. However, visualization may be better in obese patients using a curvilinear 

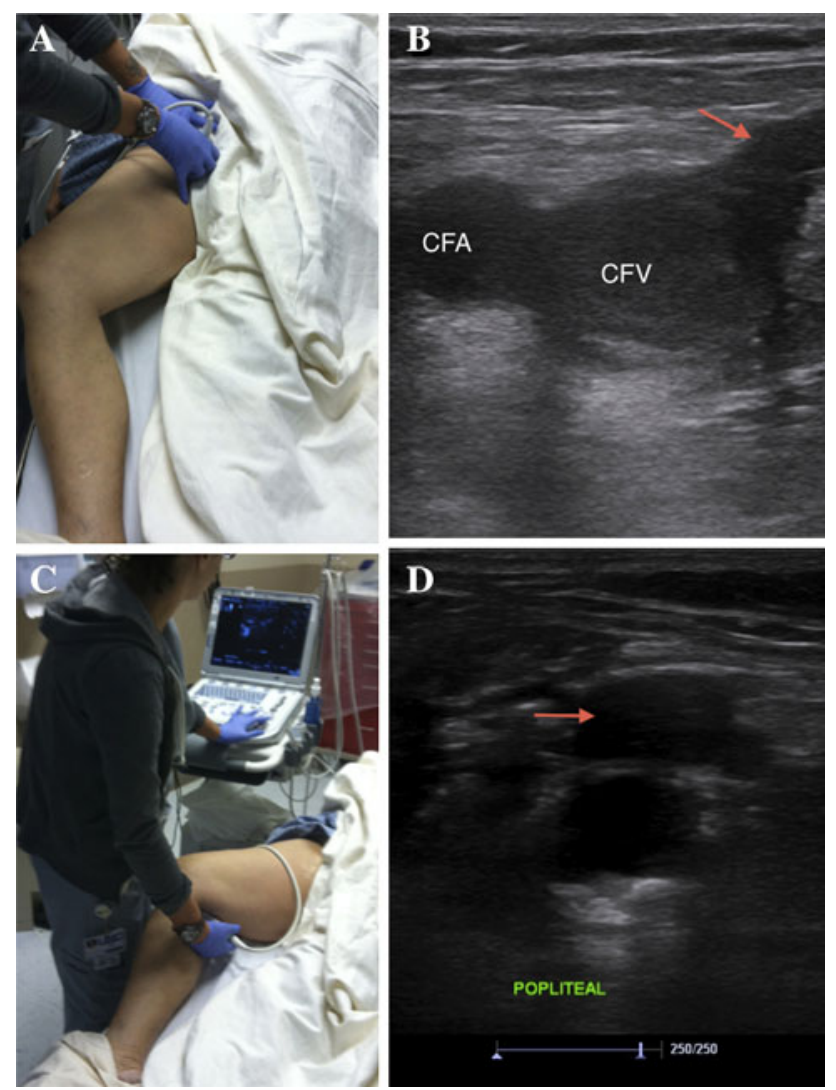

Fig. 1 a Placement of the patient in the frog leg position with probe prependicular to skin, marker to patient's right, just inferior to the inguinal ligament. b Common femoral artery vein and saphenofemoral junction (arrow). c Probe placement over the popliteal fossa. d Popliteal artery and vein (arrow)

probe as it uses a lower frequency and will give better penetration.

Place the patient in slight (about 30 degrees) of reverse Trendelenburg. This should cause pooling of blood in the veins of the lower extremities, which will help improve visualization of the vein. Expose the patient's entire leg up to the inguinal ligament. Externally rotate the patient's hip as much as is comfortable, and have them place their knee in slight flexion. This is also known as a frog leg position (Fig. 1).

The two main ultrasonographic methods to evaluate for DVT are compression and duplex ultrasound. The recommended technique for a bedside DVT study, is compression ultrasound (CUS). Compression ultrasound uses the fact that a vein will not compress when there is a DVT. All of the studies cited to this point that have dealt with DVT ultrasound have used some variation of compression ultrasound.

The compression technique focuses on the compressibility of the vein (Fig. 2). The specific locations of where to compress are described in the technique section below. Once the probe is in the right location, downward
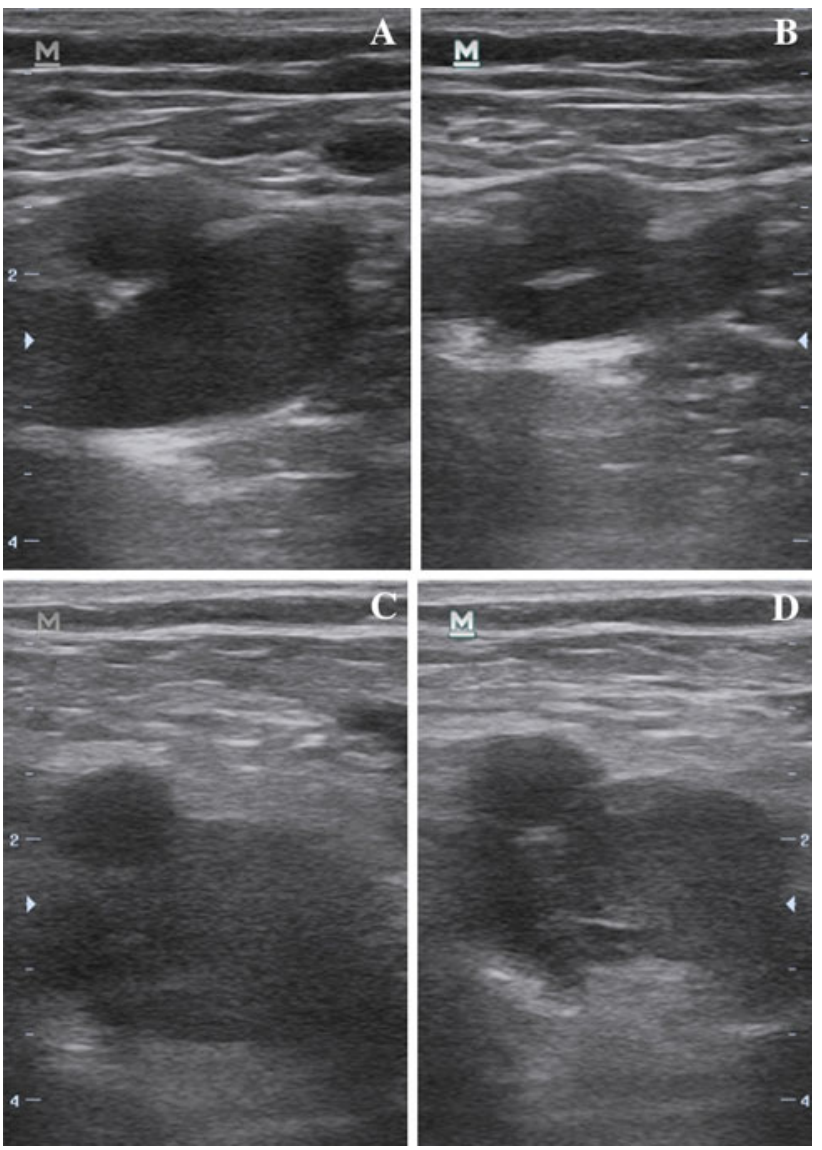

Fig. 2 a Normal hypoechoic vein. b Same vein showing compressibility. c Abnormal echogenic vein. d Same vein showing lack of compressibility

pressure is applied to the probe in an attempt to compress the underlying vein. The probe should be maintained perpendicular to the skin when compressing to get the best results. Adequate pressure is defined as enough pressure to begin to compress the adjacent artery. In all cases, a fully compressible vein is one in which no vessel lumen should be seen when adequate pressure is applied with the probe.

Some studies have used compressibility of the veins alone as the criteria for diagnosis of DVT and found good sensitivity. However, a recent study found six out of 45 positive DVT studies did not have reduced compressibility but did have visible thrombi [14••]. Thus, considering either an incompressible vein or the presence of an echogenic structure in the lumen of the vein a positive study may increase the sensitivity of the bedside scan.

Duplex ultrasonography is another option. It combines vein compression and Doppler techniques to help detect DVT. This technique is much more time consuming than CUS, and given the high sensitivity of compression ultrasound as noted in the studies above, we consider CUS the primary modality for ED and ICU physicians. 
The Two-Point Compression Technique

The two-point compression technique checks for compressibility at two locations: the femoral vein (common femoral vein) and the popliteal vein.

Begin by placing the linear probe in a transverse orientation in the groin area just below the inguinal ligament. The probe marker should be pointing to the patient's right. In this area move the probe distal along the femoral vein until the level of its confluence with the great saphenous vein. This is the first compression point. (See Fig. 1). In some cases it may be difficult to identify the great saphenous vein. When this occurs, the physician may proceed with the examination by using a point on the femoral vein just distal to the inguinal ligament.

There are several ways in which the femoral artery may be distinguished from the femoral vein. First, the artery will be only minimally compressible while the vein should be fully compressible (unless there is a DVT). Second, the vein is generally larger than the artery, and will demonstrate respiratory collapse. Third, the femoral artery is typically lateral to the femoral vein. (The mnemonic NAVeL_nerve, artery, vein, empty space, lymphatics-is often used to remember the structures in the groin from lateral to medial.) If need be, Doppler color may be used to distinguish the artery and vein. However, this requires distinguishing pulsatile flow from continuous flow, which may be difficult for the novice.

Even though the great saphenous vein is a superficial vein, a thrombus at the sapheno-femoral junction has a high chance of progression to a DVT [18] so it is important to include the sapheno-femoral junction in the view with the femoral vein as one of the two points of compression (See Fig. 1). It is recommended that thrombi in the great saphenous vein near the sapheno-femoral junction be treated with anticoagulation [19].

The second point of the two-point compression technique is the popliteal vein in the popliteal fossa (Fig. 1). Place the probe in a transverse plane, and distinguish the popliteal vein from the popliteal artery using the techniques described above for distinguishing artery and vein. Check for compressibility and the presence of an echogenic structure within the lumen of the popliteal vein in the popliteal fossa. If the physician is unable to access the patient's popliteal fossa with the patient in the position described above (leg externally rotated and knee slightly flexed), the patient may be placed in the decubitus position, prone position, or in a seated position with the legs hanging over the bed.

The Three-Point Compression Technique

This technique involves compression of the common femoral vein and popliteal vein as in two-point compression but also includes testing for compressibility of the superficial femoral vein. As mentioned above, the superficial femoral vein is actually the distal part of the femoral vein, and so even though it is called "superficial," it is a deep vein. The superficial femoral vein is found by following the common femoral vein distally until it disappears into the adductor canal. This is the third compression point.

Although both the three-point compression and twopoint compression techniques are adequate, the two pointcompression technique is easier and faster, and the current data does not suggest that the three-point compression technique has a higher sensitivity [14॰•]. More research is needed to conclusively say which technique is better.

\section{Complete Proximal Leg Compression Technique}

Some physicians recommend compressing every $1-2 \mathrm{~cm}$ along the entire visible length of the femoral vein and popliteal vein. This technique logically seems as though it would discover more clots since a greater portion of the deep veins is tested for compressibility, but data suggest that more limited examinations, including the two-point and three-point compression techniques, have very high sensitivities (approaching 100\%) [14••]. The complete proximal leg compression technique is also more time consuming than the two-point or three-point compression techniques.

\section{The Whole Leg Compression Technique}

This technique involves compression of the calf veins in addition to the whole course of the femoral and popliteal veins. This technique takes much longer than the two-point or three-point compression techniques, but it has the advantage of detecting calf-vein thrombosis. However, the clinical significance of calf-vein thrombosis is questionable. (See section on calf-vein thrombosis below.)

\section{Pitfalls and Tips}

\section{DVT Location}

Compression ultrasonography performed at the bedside does not detect abdominal or pelvic DVT, but neither do formal radiology department performed DVT ultrasound studies. As previously mentioned, ultrasonography performed by the radiology department that involve whole leg compression can find calf vein thrombosis while the two and three-point compression techniques will not. It's possible that a limited bedside ultrasound with only two or three points of compression might miss some DVTs that 
would be caught if the ultrasonographer compressed the whole leg. However, previous studies have shown that twopoint compression is nearly as sensitive for DVT as a complete ultrasound of the deep venous system from the inguinal ligament all the way to the calf $[14 \bullet \cdot, 20]$.

\section{Baker's Cysts and Lymph Nodes}

A Baker's cyst or lymph nodes can be mistaken for a DVT. Make sure to identify landmarks such that the vein is always next to the artery. When the physician is unable to identify key landmarks, something may be amiss. On ultrasound, normal lymph nodes are hypoechoic oval structures with sharp borders and a hyperechoic center, similar to a bull's-eye. A Baker cyst on ultrasound appears as an anechoic mass with sharply defined borders. If the Baker's cyst is complex, it may have internal echoes within the mass. Both a lymph node and a baker's cyst will appear ovoid with defined edges in both the longitudinal and transverse view. A vein will look ovoid only in the transverse view and will be cylindrical without borders on a longitudinal view.

\section{Acute Clots Versus Chronic Clots}

It's important to note that compression ultrasound may continue to be abnormal in up to $70 \%$ of patients within one year after DVT [21]. A positive compression ultrasound on a patient with a DVT within the last year may simply represent a chronic clot.

The age of the thrombus may be inferred from the echogenicity of the clot. Older clots appear more echodense [22]. However, this is an advanced skill that should generally be left to radiologists.

In situations when there is uncertainty of a Baker's cyst, abnormal anatomy, or when there is concern about a chronic versus acute DVT, a formal ultrasound study is recommended.

\section{Calf Vein Thrombosis}

The clinical significance of isolated calf vein thrombosis is unclear. Although calf-vein thrombosis may progress to proximal DVT, some studies have shown that anticoagulation is not beneficial in these patients [23]. One review article found no statistically significant difference for the 3-month thromboembolic rate for ultrasounds that included proximal veins only to those that included the proximal veins and calf veins (95\% CI: $0.4-0.9 \%$ vs. $0.1-0.6 \%$ ). In this study, calf vein thrombosis was treated with anticoagulation, so the authors felt treating these calf vein thromboses may represent overtreatment [24].
The American College of Emergency Physicians currently recommends that patients with a calf vein thrombosis get a repeat ultrasound in 5-7 days to evaluate for propagation into the deep venous system, which would then require anticoagulation [25•].

\section{Duplication of the Venous System}

Venous duplication of the lower extremities occurs in about $10 \%$ of patients [26]. In these patient it is important to evaluate for DVT in both venous systems. If you only evaluate for DVT in the main vein, you may miss a clot in the duplicate system, which could than propagate to the lung.

\section{Other Tips}

- If there appears to be some slight echogenicity in the lumen of the vein that you think is a clot, try adjusting the gain before concluding that it is a DVT as it may be just an artifact that will disappear when the gain is increased.

- Venous stasis may also cause some echogenicity in the lumen of the vein. Venous stasis can be differentiated from a clot by the fact that the echogenicity of stasis will be dynamic while the echogenicity of a clot should not have motion. Furthermore, a vein with venous stasis should be collapsible.

- Keep the probe perpendicular to the skin when compressing veins. This will ensure you are applying pressure directly on the vein during compression.

- If a vein has an area that looks concerning for a DVT, try turning the probe into a longitudinal orientation. This will provide a different view of the vein that may make it more clear if there is in fact a DVT.

\section{Suggested Management}

The American College of Emergency Physicians (ACEP) guidelines suggest that it is safe to anticoagulate a patient with a positive bedside compression ultrasound study. ACEP also recommends that patients get a repeat ultrasound in 5-7 days if a thrombus was found distal to the popliteal vein, patients with a high pretest probability and negative ultrasound, and those with a positive D-dimer and negative ultrasound. A DVT can be safely excluded with a negative D-dimer and a negative ultrasound without need of a repeat ultrasound [25•].

Data to support ACEP's recommendations that a negative compression ultrasound combined with a negative D-dimer can safely exclude DVT are supported by a study 
which found that only one of 598 patients with a negative compression ultrasound and negative D-dimer developed a venous thromboembolic complication at 3-months follow up [27].

In addition to use for diagnosis of DVT in a stable patient, bedside ultrasound may be useful in code situations to suggest pulmonary embolism as the cause for the cardiac arrest. Or, it may be used to evaluate for pulmonary embolism in patients who are unable to undergo CT or V/Q scan.

\section{Conclusion}

Compression ultrasound is an emerging tool that emergency and critical care physicians can use to rapidly diagnose DVT at the bedside. A bedside compression ultrasound is easy to learn and takes only a few minutes to perform. While further time and data will likely be needed before these bedside studies can replace formal studies, the evidence and trends are rapidly growing in this regard. We suggest that all emergency medicine and critical care physicians learn how to perform a bedside DVT ultrasound in order to better care for their patients.

\section{References}

Papers of particular interest, published recently, have been highlighted as:

- Of importance

•- Of major importance

1. Spencer F, Emery C, Lessard D, et al. The Worcester venous thromboembolism study: Apopulation-based study of the clinical epidemiology of venous thromboembolism. J Gen Intern Med. 2006;21(7):722-7.

2. Anderson FA, Wheeler HB, Goldnerg RJ, et al. A populationbased perspective of the hospital incidence and case-fatality rates of deep vein thrombosis and pulmonary embolism. The Worcester DVT Study. Arch Intern Med. 1991;151(5):993-8.

3. Calder K, Herbert M, Henderson SO. The mortality of untreated pulmonary embolism in emergency department patients. Ann Emerg Med. 2005;45:302-10.

4. Goodacre S, Sutton AJ, Sampson FC. Meta-analysis: the value of clinical assessment in the diagnosis of deep venous thrombosis. Ann Intern Med. 2005;143(2):129.

5. Wells et al. Evaluation of D-dimer in the diagnosis of suspected deep-vein thrombosis. N Engl J Med. 2003;349:1227-35.

6. Hull R, Hirsch J, Sackett DL, et al. Clinical validity of a negative venogram in patients with clinically suspected venous thrombosis. Circulation. 1981;64(3):622.

7. Lensing AW, Prandoni P, Brandjes D, et al. Detection of deepvein thrombosis by real-time B-mode ultrasonography. N Engl J Med. 1989;320(6):342.

8. Theodorou SJ, Theodorou DJ, Kakitsubata Y. Sonography and venography of the lower extremities for diagnosing deep vein thrombosis in symptomatic patients. Clin Imaging. 2003;27(3):180-3.
9. Cogo A, Lensing AW, Prandoni P, et al. Comparison of real-time B-mode ultrasonography and Doppler ultrasound with contrast venography in the diagnosis of venous thrombosis in symptomatic outpatients. Thromb Haemost. 1993;70(3):404-7.

10. - Kory PD, Pellecchia CM, Shiloh AL, et al. Accuracy of ultrasonography performed by critical care physicians for the diagnosis of DVT. Chest. 2011;139(3):538. This study showed that critical care physicians who generally don't use ultrasound for diagnosis as frequently as emergency physicians also can perform bedside ultrasound to diagnose DVT with good accuracy.

11. Theodoro D, Blaivis M, Duggal S. Real-Time B-mode ultrasound in the ED saves time in the diagnosis of Deep Vein thrombosis DVT. Am. J. Emerg. Med. 2004;22(3):197-200.

12. Jacoby J, Cesta M, Axelband J. Can emergency medicine residents detect acute deep venous thrombosis with a limited, twosite ultrasound examination? J Emerg Med. 2007;32(2):197-200.

13. Frazee BW, Snoey ER. Levitt. Emergency department compression ultrasound to diagnose proximal deep vein thrombosis. J Emerg Med. 2001;20(2):107-12.

14. •• Crisp JG, Lovato LM, Jan TB. Compression ultrasonography of the lower extremity with portable vascular ultrasonography can accurately detect deep venous thrombosis in the emergency department. Ann. Emerg Med. 2010;56(6):601-610. This is a recent study that provides some of the strongest evidence in favor of bedside two-point compression ultrasound for diagnosis of DVT.

15. Kline JA, O’Malley PM, Tayal VS, et al. Emergency clinicianperformed compression ultrasonography for deep venous thrombosis of the lower extremity. Ann Emerg Med. 2008;52:437-45.

16. Burnside P, Brown M, Kline J. Systematic review of emergency physician-performed ultrasonography for lower-extremity deep vein thrombosis. Acad Emerg Med. 2008;15:493-8.

17. Scarvelis D, Wells P. Diagnosis and treatment of deep-vein thrombosis. CMAJ. 2006;175(9):1087-92.

18. Chengelis DL, Bendick PJ, Glover JL, et al. Progression of superficial venous thrombosis to deep vein thrombosis. J Vasc Surg. 1996;24(5):745.

19. Litzendorf ME, Satiani B. Superficial venous thrombosis: disease progression and evolving treatment approaches. Vasc. Health Risk Manag. 2011;7:569-75.

20. Pezzullo JA, Perkins AB, Cronan JJ. Symptomatic deep vein thrombosis: diagnosis with limited compression US. Radiology. 1996;198(1):67-70.

21. Prandoni P, Cogo A, Bernardi E, Villalta S. A simple ultrasound approach for detection of recurrent proximal-vein thrombosis. Circulation. 1993;88(4 Pt 1):1730.

22. O'Shaughnessy AM, FitzGerald DE. Organization patterns of venous thrombus over time as demonstrated by duplex ultrasound. J. Vasc. Invest. 1996;2:75.

23. Schwarz T, Buschmann L, Beyer J, et al. Therapy of isolated calf muscle vein thrombosis: a randomized, controlled study. J Vasc Surg. 2010;52(5):1246.

24. Righini M, Paris S, Le Gal G, Laroche JP, et al. Clinical relevance of distal deep vein thrombosis. Review of literature data. Thromb Haemost. 2006;95(1):56-64.

25. - Rios M, Lewiss R, Saul T. Focus on: emergency ultrasound for deep vein thrombosis. Retrieved from http://www.acep.org/, 2009. These are the most recent guidelines published by one of the leading societies of emergency medicine physicians.

26. Simpson WL, Krakowsi DM. Prevalence of lower extremity venous duplication. Indian J. Radiol Imag. 2010;20:230-4.

27. Bernardi D, Prandoni P, Lensing AW. D-dimer testing as an adjunct to ultrasonography in patients with clinically suspected deep vein thrombosis: prospective cohort study. The Multicentre Italian D-dimer Ultrasound Study Investigators Group. BMJ. 1998;317(7165): 1037 . 